\title{
Development Opportunity and Development Direction of Vocal Music Teaching Mode in Diversified Era
}

\author{
Han Jihong ${ }^{1, a}$ \\ ${ }^{1}$ Linyi University,Yishui,Shandong,276400 \\ aLydxhjh@163.com
}

Keywords: vocal music; diversification; development

\begin{abstract}
In the diversified era, the diversification ideas is applied in the subject in practice, and education reform provides an opportunity to vocal music teaching development and reform, the development of diversified vocal music teaching mode has become the requirements of the era development, it has important function and meaning in improving the students' interest in learning, achieving individualized teaching, and deepening the curriculum reform. In the development of diversified teaching mode, we should grasp the direction of development, and teaching mode should be designed properly, so as to improve the teaching effect of vocal music, and promote the development of subject teaching.
\end{abstract}

\section{Introduction}

The vocal music is referred as a special subject, and it has very important function and meaning in the cultivation of students' cultural accomplishment, appreciation, intellectual and psychological quality. At the same time, vocal music is as a technical subject, in the teaching of vocal music, the method of singing, vocal teaching mode, and teaching method and means are important, these methods should also adapt to the trend of the times. At present, due to many factors, and the traditional music teaching models are difficult to adapt to the development needs of the times, and different problems in teaching and training are highlighted. With the arrival of the diversified era[1], the education reform and college informatization construction provide an opportunity for the development of vocal music teaching. In this situation, the vocal music teaching should keep pace with the times, actively innovation should be taken into consideration, and the development of diversified teaching mode is carried out. In this paper, the reforming methods are researched, and development opportunity and development direction of vocal music teaching mode in diversified era are analyzed, and the relative reform measures are proposed, so as to improve the teaching effect of vocal music[2], and promote the development of subject teaching. The research results show good and important value in practice.

\section{Opportunities for the development of vocal music teaching ion diversified era}

A. Traditional single teaching mode has many disadvantages

Expression of human feelings is the main features of the music, but in the traditional teaching mode, it focus on only theoretical knowledge, the students desire expression and release of feelings are neglected. The students' main body function is ignored. It is difficult to stimulate the potential of students with the traditional teaching mode. Secondly, the vocal music teaching mode in China tends to emphasize systematic and integrity of the vocal music specialized knowledge, students mental perception is less concerned about. Some important items such as cultural understanding, environment cognition, and cognitive expertise are ignored. In this teaching mode, it is easy to cause the students have a strong theoretical knowledge, but the music artistic conception is hard to be grasped correctly. Vocal music is the art with strong technology and practice performance, the vocal music practice course plays an important role in improving students' vocal skills[3], but the traditional single teaching mode pays more attention to professional theoretical knowledge in teaching. In the curriculum arrangement, professional theory courses and general cultural curriculums have taken a large proportion, but the practical curriculum is less. It has not benefits in 
cultivating the students' practical ability, the existing defects of traditional teaching mode directly affects the vocal music teaching quality, it has also required the vocal music teaching mode must be reformed, and the development of vocal music teaching can be obtained in further with the reform of teaching mode[4].

B. New curriculum standard and requirements of quality education

In the new "full-time compulsory education standards of music course", emphasizing the aesthetic experience is the core of vocal music education, in the vocal music teaching, teaching contents should be lively and interesting, the rich and colorful teaching modes should be fused. The vocal music teaching should pay attention to the main role of students, respect for different individual with different music experience, we should advocate in establishing a diversified concept. The quality education is put forward for the exam oriented education, so the talents in quality education have the rational quality emotional quality, emotion quality and quality of body and mind. This requires that the process of teaching should pay attention to cultivate the emotion, and the culture emotion promotion should be promoted. We should pay attention to the personalized development, so that the four kinds of qualities of students can obtain full and comprehensive development. The new curriculum standard and the requirement of quality education emphasize the necessity of reform of vocal music development, and it also points out the direction for the development vocal music teaching[5].

C. Initial formation of diversified teaching system in China

With the arrival of the diversified era, the diversification concept has penetrated into every aspect of teaching, the practical application of diversification concept has made the diversified teaching system has been initially formed, including the diversification of teaching content, teaching methods, diversification evaluation, and diversified teaching modes etc. The diversified teaching system of various subjects is formed, and it can provide a reference to the diversified development of the vocal music teaching mode.

D. College informatization construction provides the basis for the diversification education

With the development of computer technology and information technology, information construction level of colleges and universities has made gradual improvement, and it promotes the diversified reform in teaching environment, teaching methods, teaching evaluation, the network and transformation reform is developed, the rich and broad network world has provided the development basis of teaching environment, and the multi forms of communication, ultra realistic operation function have supported the close exchange of teaching and learning from the technology, it can excite the innovation of teaching and learning fundamentally, and promote the scientific development of education.

\section{Significance of developing the diversified vocal music teaching mode in diversified era}

\section{A. Teach students in accordance with their aptitude is realized}

There is a discrepancy between the individual cognitive level, learning ability, the quality of students is different, in the vocal music teaching, some students professional basis are better, and vocal condition is good, but some of the students professional foundation is weak. In the practice of teaching condition, according to the professional basis of students, targeted to develop teaching repertoire, the theoretical knowledge and singing skills are trained are improved individually, the diversified teaching mode is drafted for different characteristics of students, teachers can carried out the teaching works according to vocal techniques, singing, vocal learning ability of different students, targeted teaching content and implementation plans are obtained. We can teach students in accordance with their aptitudes, and the teaching effect and quality will be improved as result.

B. Help to improve the students' interest in learning

From the perspective of educational psychology, the interest is a kind of inner strength to push people to seek knowledge. In the vocal music teaching, the learning interest of the students has the fundamental advantage which is unable to substitute other factors by other factors. It not only affects the quality and efficiency of vocal music teaching, but also stimulates students' potential in learning. So the students' interest in learning has important significance. We should pay attention to 
help the students to improve the interest in learning. In the cultivation of interest in learning, the same teaching contents with different teaching methods will have different effects. On the contrary, the different teaching content with the same teaching method to inspire student interest is different. The vocal music teaching mode of diversity can meet interest demand of students from different point of views, so as to improve the students' interest in learning

C. Conducive to deepening the curriculum reform

Teaching reform is a two-way interaction, it is not only the mission of teacher, it also needs student feedback in the effect of the reform. In current vocal music teaching reform, due to the existence of some problems, due to the existence of some problems, the actual effect of teaching reform is difficult to improve, vocal music teaching reform facing the "bottleneck", so it is not conducive to the further deepening reform. The advantage of diversified teaching mode is shown in stimulating the interest of students in terms of development, it can promote teaching reform to break the bottleneck, deepen the curriculum reform.

\section{Development direction of vocal music teaching diversification mode}

A.Using hierarchical teaching mode

According to knowledge level, learning ability and the potential ability, the hierarchical teaching mode is designed, students can be divided into different levels, and classification guidance is taken. It is one of the most representative teaching modes in the diversified era at present, and it has been widely used in the domestic teaching of various subjects. Hierarchical teaching mode admits the individual differences of students, there are differences in students' ability, intelligence and other aspects of the existence factors. So we should teach students in accordance with their aptitude, and develop targeted teaching plan, meet the diverse ideas. In the vocal music teaching, the hierarchical teaching is used, and a certain hierarchical form is taken. According to the student's potential, ability and level, combined with different arrangement of teaching contents, the students are divided into different levels in hidden partition. The team teaching is carried out. With this method, the personality differences of students are respected, teaching resources are saved, and teaching efficiency is improved. According to the hierarchical teaching of vocal music teaching, the students are layered in different levels according to the actual situation of the student, the prepare lessons layering, targets layering and evaluation layering are obtained. According to the vocal music teaching syllabus, teaching materials are layered based on the students' cognitive ability, different goals are established. According to hierarchical and teaching content, the creative performance evaluation is required by the students with good foundation and good condition. For a high level of teaching evaluation, the students' potential is stimulated, for the relatively low level students, the incentive evaluation are implemented, so that different levels of students can make progress to a certain degree.

B. Pay attention to emotion education

Emotional experience is an important factor in influencing the singer voice quality, action expression and the other effect of the performance. The singer creativity has the important influence on the sound quality, and the rich emotion experience can stimulate individual performance, so as to embody the connotation and emotional repertoire together. The vocal music teaching needs to pay attention to the cultivation of students' emotional experience and emotional teaching. Emotional experience is obtained by the direct and indirect methods, wherein, their life experience is a direct way to obtain the emotional experience, so in the teaching mode, teachers should guide students to observe, to experience life in the natural scenery and life picture, and capture the exciting, poetic paintings of scenes in life, enrich their life accumulation constantly. As a person, emotional experience from their own experience is limited, at the same time in the expression of musical works, it requires students to have better emotional awareness. The experience nature, extent, change of the emotional state of the amplitude and intensity can be accurate experienced. So teaching mode should pay more attention to indirect means in cultivating students' emotional experience. The references such as drama, film, poetry, literature and other aspects of information are important resources, these information can guide students to realize all kinds of feelings in life, 
so that students can have rich emotional experience. The emotional foundation is constructed, and the students can grasp the emotion feature in concert works, then the singer can express the emotion in singing, the singing has more authenticity.

C. Strengthen cultural cultivation teaching

The profound cultural literacy can cultivate students' aesthetic feeling, make students better understand in singing to the content of the songs, and the emotion of the creation can be grasped. The students writing level is improved. Therefore, teachers should also emphasize the training of students' cultural accomplishment in the diversification of teaching, enhance students' cultural accomplishment. The vocal music course requires students to have the cultural accomplishment and cultivation except for the vocal music skills of professional phase history and culture, but also requires students should have social science and natural science. Some of the basic knowledge is important. In the vocal repertoire of practice, teachers should provide students with related cases about music, combining culture and emotion experience and training, and guide students to clear the importance of culture in vocal music learning. We can lead the students to visit the local music art exhibition, let the students stay in strong cultural atmosphere with the infection in the music, at the same time the culture cultivation education must be lively and vivid, to improve the learning interest of the students.

D. Proper use of digital technology

With the development of information technology, and its application in the field of education, the development of education is promoted. In the vocal music teaching, teachers should realize the interactive teaching of vocal music in the positive application of computer technology, computer training is taken, and the students participate in the process of performance. According to the concert pitch, timbre, volume, the real-time feedback is obtained. It can let the student computer prompt in real time, the improvisation is carried out, and the vocal skills of students are fully exercised. The digital technology is used in the teaching application, we should pay attention to the students are learning objective, the digital technology is a kind of auxiliary tool, it serves the students, and serve the teaching. The appropriate use of digital technology in the teaching should be gasped, to avoid the abuse of the technology.

\section{Conclusions}

In diversified era, through continuous exploration and innovation, new teaching patterns and modes of vocal music teaching are emerged. In vocal music teaching mode, different teaching modes are not isolated, but interrelated. The interaction of teaching mode is researched. In the teaching practice, the development direction of diversified teaching mode should be hold. The development direction of diversified teaching mode is important to be researched in education reform. The education reform provides an opportunity to vocal music teaching development and reform, the development of diversified vocal music teaching mode has become the requirements of the era development, it has important function and meaning in improving the students' interest in learning. In this new mode, the enthusiasm of the students are mobilized comprehensively, the students' innovation ability and practice ability are improved. Thus the teaching effect is improved, and the development of the vocal music teaching is promoted.

\section{References}

[1] Han Mengjie, Zhang Dexiang. On Market Mechanism of Structural Changes in American Higher Education[J].Educational research, 2014,1:124-131.

[2] Yu Jia, Zhu Zhiqiang. Research On I-U-R Collaborative Innovation Based on Ecological Theory[J]. Bulletin of Science and Technology, 2013, 29(7): 225-230.

[3] Chen Guocai. Research on SilverLight Based WebGIS System and Its Application[J]. Bulletin of Science and Technology, 2013, 29(4): 177-179.

[4] QIU Jing, WANG Ping. Encryption Algorithm for Compressed Image Based on Chaotic Maps[J]. Computer Science, 2012, 39(6): 44-46.

[5] MING LI, KAI ZENG, WENJING LOU. Opportunistic Broadcast of Event-Driven Warning Messages in Vehicular Ad Hoc Networks with Lossy Links[J]. Computer Networks, 2011,55(10):2443-2464. 